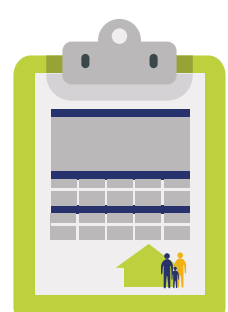

\title{
PROPOSTA DE UM INSTRUMENTO DE AUTOAVALIAÇÃO PARA MELHORIA DO ACOMPANHAMENTO DAS CONDICIONALIDADES DO PROGRAMA BOLSA FAMÍLIA NA ESTRATÉGIA SAÚDE DA FAMÍLIA
}

\author{
PROPOSAL FOR A SELF-EVALUATION INSTRUMENT FOR IMPROVING THE \\ ACCOMPANYING OF THE CONDITIONALITIES OF THE FAMILY GRANT \\ PROGRAM IN THE FAMILY HEALTH STRATEGY
}

\section{Ricardo Henrique Vieira de Melo}

Mestre em Saúde da Família (RENASF/UFRN), preceptor do Pet-Saúde (UFRN), preceptor da Residência Multiprofissional em Saúde (UFRN), dentista da Estratégia Saúde da Família de Natal (RN).

E-mail: ricardohvm@gmail.com

Márcia Cunha da Silva Pellense

Mestre em Saúde da Família (RENASF/UFRN), enfermeira, especialista em Gerenciamento da Atenção Básica, secretária adjunta de Atenção Integral à Saúde da Secretaria Municipal de Saúde de Natal (RN).

E-mail: marciapellense@gmail.com

Marise Soares Almeida

Mestre em Saúde da Família (RENASF/UFRN), enfermeira da Estratégia Saúde da Família da Secretaria Municipal de Saúde de Natal (RN). E-mail: marise-sa@hotmail.com

\section{Andrezza Karine Araújo de Medeiros Pereira}

Mestre em Saúde da Família (RENASF/UFRN), enfermeira, docente da Universidade Estadual do Rio Grande do Norte (UERN). E-mail: andrezza_kam@hotmail.com

Marsilene Gomes Freitas

Mestre em Saúde da Família (RENASF/UFRN), docente da Universidade Federal do Rio Grande do Norte

(FACISA/UFRN) e Universidade Potiguar (UNP), médica pediatra, supervisora do PROVAB (MS/BR).

E-mail: marsilenegomes@gmail.com

\section{Neuma Marinho de Queiroz Costa Cunha}

Mestre em Saúde da Família (RENASF/UFRN), docente da Universidade Federal do Rio Grande do Norte (UFRN) e Universidade Potiguar (UNP), supervisora do PROVAB (MS/BR), médica da Estratégia de Saúde da Família de Natal (RN). E-mail: neuma.ufrn@gmail.com

Rosana Maria Ferreira de Moura Lima

Mestre em Saúde da Família (RENASF/UFRN), especialista em Saúde Pública e Nutrição Social, nutricionista da Secretaria de Estado da Saúde Pública do Rio Grande do Norte (SESAP). E-mail: rosanaml@oi.com.br

\section{Paulo de Medeiros Rocha}

Doutor em Saúde Coletiva (Instituto de Medicina Social/UERJ), pós-doutorado (Instituto de Medicina

Preventiva/Universidade de Lisboa/Portugal), docente do Departamento de Saúde Coletiva da Universidade Federal do Rio Grande do Norte (UFRN). E-mail: paulodemedeirosrocha@gmail.com

\section{Severina Alice da Costa Uchoa}

Doutora em Saúde Coletiva (Instituto de Medicina Social/UERJ), docente do Departamento de Saúde Coletiva da Universidade Federal do Rio Grande do Norte (UFRN). E-mail: aliuchoa@hotmail.com 


\section{RESUMO}

O Programa Bolsa Família está inserido no contexto das estratégias políticas adotadas para reduzir as desigualdades sociais e de renda no Brasil. O setor de saúde tem importante papel na melhoria da qualidade de vida das famílias beneficiadas pelo programa através do acompanhamento das condicionalidades de saúde. Objetiva-se, com o presente artigo, relatar a experiência de uma prática em comunidade que teve como meta apresentar um instrumento de autoavaliação para melhoria do acompanhamento das condicionalidades do Programa Bolsa Família na Estratégia Saúde da Família. Como metodologia, foram utilizadas a análise documental e a aplicação de uma ferramenta de autoavaliação elaborada a partir do instrumento de autoavaliação do Programa Nacional de Melhoria do Acesso e da Qualidade da Atenção Básica (AMAQ/AB). Os sujeitos foram os agentes comunitários de saúde de uma unidade da Estratégia de Saúde da Família do Município de Natal (RN). A construção dos dados ocorreu durante três momentos: sensibilização e apresentação dos relatórios consolidados do Programa Bolsa Família; leitura e aplicação do instrumento de autoavaliação; e análise dos resultados. Quantos aos resultados, os achados foram consonantes com os relatórios consolidados do Sistema de Informação do Programa Bolsa Família. Os autores apresentaram um instrumento pertinente de autoavaliação com potencial de ampliação nas demais unidades de saúde para auxiliar a gestão local no monitoramento das condicionalidades da saúde. Conclui-se que a vivência contribuiu para aumentar o conhecimento dos participantes para a reflexão sobre seus processos de trabalho e para o aprimoramento de habilidades e competências autoavaliativas. Desse modo, apontou-se a necessidade de realização de estudos complementares de natureza qualitativa para aprofundamento dos aspectos abordados.
PALAVRAS-CHAVE: Programas de Autoavaliação. Programa Bolsa Família. Estratégia Saúde da Família.

\section{ABSTRACT}

Family Grant Program is inserted in the context of the political strategies adopted to reduce social and income inequalities in Brazil. The health sector plays an important role in improving the quality of life of families benefited by the program through the monitoring of health conditionalities. Objective: This article reports the experience of a practice in the community that aimed to present a self-assessment tool to improve the follow up of the conditionalities of the Family Grant Program in the Family Health Strategy. Methods: Document analysis and the application of a self-assessment tool elaborated using the Self-Assessment tool of the National Program for Improving Access and Quality of Primary Care (AMAO / AB) were used. The subjects were the Community Health Agents of a Family Health Strategy Unit of the Municipality of Natal (RN). The construction of the data took place during three moments: awareness and presentation of the consolidated reports of the Family Grant Program, reading and application of the self-assessment instrument and analysis of the results. Results: The findings were consonant with the consolidated reports of the Family Grant Program Information System. It presented a pertinent self-assessment tool with potential for expansion in other health units to assist local management in monitoring health conditionalities. Conclusions: The experience contributed to increase the knowledge of the participants for the reflection on their work processes and for the improvement selfassessment skills. He pointed out the need for complementary studies of a qualitative nature to deepen the aspects covered.

KEYWORDS: Self-Evaluation Programs. Family Grant Program. Family Health Strategy. 


\section{INTRODUÇÃO}

O Programa Bolsa Família (PBF) se apresenta com uma lógica focalizada, porém integrada a outras políticas públicas universalizadas, que envolvem a saúde e a educação. Possui também objetivos ambiciosos de articulação de serviços sociais e assistenciais, e busca beneficiar uma população carente através da transferência e redistribuição de renda. $\bigcirc$ amadurecimento institucional do programa através de diversas normatizações, juntamente com uma maior centralização de decisões por parte do Governo Federal, vem contribuindo para a sua consolidação no país, bem como para um maior controle das condicionalidades de saúde e educação, visando fomentar o acesso das populações mais vulneráveis às políticas sociais tradicionais (SOUZA; MARIN, 2017).

O PBF está inserido no contexto das estratégias políticas adotadas pelo Governo Brasileiro, ao longo das últimas décadas, visando reduzir as desigualdades sociais e de renda em nosso país, tendo como objetivos: promover o acesso a uma rede de serviços públicos relacionados às condicionalidades do PBF; combater a fome, objetivando o alívio imediato de famílias em situação de vulnerabilidade; minimizar a pobreza e alcançar a Segurança Alimentar e Nutricional (SAN) da população; estimular a emancipação das famílias que vivem em situação de pobreza e extrema pobreza; e promover a intersetorialidade de ações sociais do poder público (BRASIL, 2010).

Criado em 2004, pela Lei $n^{\circ} 10.836$, o PBF integra o Plano Brasil Sem Miséria e está vinculado ao Ministério do Desenvolvimento Social e Combate à Fome (MDS), sendo uma das prioridades do Governo Federal para a área social. Trata-se, portanto, de um programa governamental de transferência de renda com condicionalidades, que beneficia famílias em situação de pobreza e extrema pobreza.
As famílias, para receberem a transferência de renda mensalmente, devem estar inscritas no Cadastro Único e assumir os compromissos previstos entre as condicionalidades do Programa, as quais incluem as áreas da Saúde, Educação e Assistência Social. Essas condicionalidades referem-se aos compromissos assumidos, tanto pelas famílias inscritas no PBF, quanto pelo Poder Público, para ampliação do acesso a direitos sociais básicos. As famílias devem assumir e cumprir esses compromissos para continuarem no PBF, e o Poder Público deve se responsabilizar pela oferta dos serviços públicos de saúde, educação e assistência social (BRASIL, 2012).

$\mathrm{Na}$ área da saúde, as famílias devem cumpriro calendáriovacinal e o acompanhamento do Crescimento e Desenvolvimento (CD) das crianças menores de 7 anos; e as mulheres na faixa de 14 a 44 anos que estiverem gestantes ou nutrizes devem realizar o pré-natal e o acompanhamento da sua saúde e da saúde do bebê. Ressalta-se que, a partir da $2^{a}$ vigência de 2012, foram disponibilizadas no Sistema de Gestão do PBF na Saúde também as informações sobre mulheres com idade entre $7 \mathrm{e}$ 13 anos e acima de 44 anos. Essa medida teve o objetivo de possibilitar a eleição dessas mulheres ao Benefício Variável à Gestante (BVG), ao se tornarem grávidas. O BVG integra o conjunto de benefícios variáveis do PBF e é pago pelo Ministério do Desenvolvimento Social e Combate à Fome às famílias beneficiárias que tenham gestantes em sua composição. Para tornar a beneficiária elegível ao recebimento do BVG, é necessário que o serviço de saúde municipal indique a situação gestacional no referido Sistema. Entretanto, o público de acompanhamento obrigatório permanece sendo composto por mulheres entre 14 e 44 anos, e crianças menores de 7 anos (CABRAL et al., 2013).

O Poder Público deve fazer o acompanhamento gerencial dessas condicionalidades, identificando os motivos 
do não cumprimento por parte das famílias. A partir daí, são implementadas ações de acompanhamento das famílias em descumprimento. Na saúde, esse acompanhamento é feito por meio do registro das informações em instrumento próprio (mapa) do Ministério da Saúde, as quais deverão ser inseridas no Sistema de Informação do Programa. As famílias que descumprem as condicionalidades estão sujeitas a penalidades que vão de advertência à suspensão temporária do benefício e até cancelamento deste (CARVALHO; ALMEIDA; JAIME, 2014).

Portanto, para a superação da pobreza, - PBF pressupõe intervenções sociais que possibilitem a articulação de três dimensões essenciais, tendo o setor saúde um importante papel nesse contexto: promoção do alívio imediato da pobreza, através da transferência direta de renda; reforço ao exercício de direitos sociais básicos nas áreas da Saúde, da Educação e da Assistência Social, por meio do cumprimento das condicionalidades exigidas; e coordenação de programas complementares, a exemplo dos programas de geração de trabalho e renda, alfabetização de adultos, fornecimento de registro civil e demais documentos (GONÇALVES; MENICUCCl; AMARAL, 2017).

No âmbito do PBF, o setor saúde tem importante papel no que se refere à melhoria da qualidade de vida e de saúde das famílias inscritas no Programa. O acompanhamento das condições de saúde dos participantes do PBF foi acordado no Pacto pela Vida, em 2006, cabendo às equipes de saúde da atenção básica a responsabilidade, durante o período de acompanhamento ou vigência (total de meses que compreende o início e o término de cada ciclo de acompanhamento das condicionalidades), fazer a busca ativa das famílias em situação de vulnerabilidade social, garantindo a elas o acesso aos serviços de saúde e monitorando o cumprimento das condicionalidades exigidas. Destaca-se que, para que seja possível o alcance das condicionalidades de saúde, é imprescindível que os gestores e equipes informem à família sobre o papel da Saúde no cumprimento das ações, e que deixem a família ciente de suas próprias responsabilidades na melhoria de suas condições de saúde e nutrição (BRASIL, 2012).

Na atenção básica, as equipes envolvidas na oferta de ações relativas às condicionalidades são as equipes de Saúde da Família, os Núcleos de Apoio à Saúde da Família (NASF) e as equipes de Unidades Básicas de Saúde. Convém ressaltar que os agentes comunitários de saúde (ACS) têm importante papel quanto ao apoio às equipes de saúde, tendo em vista que são os profissionais que conhecem e lidam diariamente com a realidade de saúde e nutrição das famílias de um determinado território. Os ACS devem registrar os dados coletados referentes às condicionalidades de saúde dos indivíduos inscritos no PBF em formulários próprios do Programa, chamados de Mapas de Acompanhamento. Posteriormente, esses formulários devem ser inseridos, ao menos uma vez a cada vigência, no Sistema de Gestão do Programa Bolsa Família na Saúde. Ressalta-se que os dados que dizem respeito ao estado nutricional das pessoas atendidas pelo PBF são automaticamente enviados para o Sistema de Vigilância Alimentar e Nutricional Web (SISVAN - WEB) ao final de cada vigência (VASCONCELOS et al., 2017).

As condicionalidades do PBF são imprescindíveis para incentivar o acesso a serviços de saúde a famílias pobres e extremamente pobres, afim de prevenir doenças e estimular o pré-natal e a puericultura, uma vez que o PBF é um importante instrumento para atualização do cartão de imunização de crianças, adolescentes e representantes legais do benefício. A regularidade do recebimento do benefício vem contribuindo para que as famílias beneficiárias possam programar seus gastos em favor de uma melhor satisfação familiar, também favorecendo a aquisição de bens de consumo, com incentivo ao comparecimento ao 
serviço de saúde e educação, bem como ao acesso a serviços especializados de saúde e medicamentos que não estão disponíveis na rede pública de saúde (CAMPARA; VIEIRA; POTRICH, 2017).

A perspectiva de punir as famílias que não cumprem as condicionalidades parece incompatível com os objetivos de promoção social do PBF. Assim, não se pode deixar de considerar as dificuldades cotidianas de sobrevivência que as famílias vivenciam para atender às requisições impostas. Se, por um lado, as condicionalidades exigidas têm potencial para facilitar - acesso de estratos da população que dificilmente conseguiriam chegar aos serviços, por outro lado, desafiam a capacidade de absorção do aumento da demanda por parte dos serviços de saúde, testando a contrapartida da oferta de ações básicas de saúde elencadas (RIBEIRO; SHIKIDA; HILLBRECHT, 2017).

Este artigo relata a experiência de uma prática na comunidade desenvolvida por discentes do Mestrado Profissional em Saúde da Família (MPSF), coordenado pela Rede Nordeste de Formação em Saúde da Família (RENASF), na instituição nucleadora Universidade Federal do Rio Grande do Norte (UFRN), em uma Unidade de Saúde da Família (USF) do Distrito Sanitário Oeste (DSO), do Município de Natal/RN. Este trabalho teve como objetivo apresentar um instrumento de autoavaliação para melhoria do acompanhamento das condicionalidades do Programa Bolsa Família na Estratégia Saúde da Família.

\section{METODOLOGIA}

Trata-se de um relato de experiência com análise documental e aplicação de uma ferramenta de autoavaliação elaborada a partir do instrumento de autoavaliação do Programa Nacional de Melhoria do Acesso e da Qualidade da Atenção Básica (AMAQ/AB). A ferramenta AMAO foi construída após revisão e adaptação do projeto Avaliação para Melhoria da Qualidade (AMO) da Estratégia Saúde da Família e de outros instrumentos de avaliação de serviços de saúde, utilizados e validados nacional einternacionalmente, a exemplo do MoniQuor, PCATool e Quality book of Tools (BRASIL, 2017).A ferramenta foi desenvolvida com base na dimensão relacionada ao Processo de Trabalho e Atenção à Saúde do AMAQ, acrescentando (Quadro 1) uma abordagem referente às subdimensões: Ações Gerais Relacionadas ao Acompanhamento das Famílias; e Ações Relacionadas à Atuação dos agentes comunitários de saúde. A dimensão Processo de Trabalho e Atenção à Saúde consistiu na organização do serviço e estruturação dos processos de trabalho da equipe que realizava o cuidado. Por sua vez, a subdimensão Ações Gerais Relacionadas ao Acompanhamento das Famílias tratava da identificação do acompanhamento das condicionalidades da saúde. Já a subdimensão Ações relacionadas à Atuação dos agentes comunitários de saúde, buscava identificar o desempenho desses profissionais em relação ao acompanhamento das famílias do PBF. 


\begin{tabular}{|c|c|c|}
\hline DIMENSÃO & \multicolumn{2}{|c|}{ SUBDIMENSÃO: AÇÕES GERAIS NO ACOMPANHAMENTO DAS FAMÍLIAS } \\
\hline \multirow{5}{*}{$\begin{array}{l}\text { Processo de Trabalho } \\
\text { e Atenção à Saúde }\end{array}$} & \multirow{2}{*}{ Gestantes e Nutrizes } & $\begin{array}{l}\text { Fazer a inscrição no pré-natal e comparecer às consul- } \\
\text { tas na Unidade de Saúde, com o cartão da gestante, } \\
\text { de acordo com o calendário mínimo preconizado pelo } \\
\text { Ministério da Saúde. }\end{array}$ \\
\hline & & $\begin{array}{l}\text { Participar de atividades educativas ofertadas pelas } \\
\text { equipes de saúde sobre aleitamento materno e promo- } \\
\text { ção da alimentação saudável. }\end{array}$ \\
\hline & \multirow{3}{*}{$\begin{array}{l}\text { Responsáveis pelas } \\
\text { crianças menores de } \\
7 \text { anos }\end{array}$} & $\begin{array}{l}\text { Manter em dia o calendário de vacinação, conforme } \\
\text { preconizado pelo Ministério da Saúde. }\end{array}$ \\
\hline & & $\begin{array}{l}\text { Realizar o acompanhamento do crescimento e desen- } \\
\text { volvimento infantil por meio da vigilância alimentar e } \\
\text { nutricional, conforme o calendário mínimo preconizado } \\
\text { pelo Ministério da Saúde. }\end{array}$ \\
\hline & & $\begin{array}{l}\text { Participar de atividades educativas ofertadas pelas } \\
\text { equipes de saúde sobre promoção da alimentação } \\
\text { saudável. }\end{array}$ \\
\hline \multirow{5}{*}{$\begin{array}{l}\text { Processo de } \\
\text { Trabalho e Atenção } \\
\text { à Saúde }\end{array}$} & \multicolumn{2}{|c|}{ SUBDIMENSÃO: ATUAÇÃO DOS AGENTES COMUNITÁRIOS DE SAÚDE } \\
\hline & \multirow{2}{*}{$\begin{array}{l}\text { Ações relacionadas às } \\
\text { condicionalidades }\end{array}$} & Orientação das condicionalidades às famílias. \\
\hline & & Registro no mapa de acompanhamento. \\
\hline & $\begin{array}{l}\text { Ações relacionadas à } \\
\text { orientação das famílias }\end{array}$ & $\begin{array}{l}\text { Identificação das famílias. } \\
\text { Orientação às famílias sobre utilização do recurso. }\end{array}$ \\
\hline & $\begin{array}{l}\text { Ações relacionadas ao } \\
\text { controle social }\end{array}$ & $\begin{array}{l}\text { Orientação à comunidade sobre acesso, renda e con- } \\
\text { trole social. }\end{array}$ \\
\hline
\end{tabular}

Quadro 1 - Dimensões e Subdimensões da avaliação

Fonte: Adaptado com base em AMAQ-AB (2017).

Para análise do instrumento foram criados padrões de qualidade obtidos a partir de uma escala de pontuação, variando entre 0 e 10 pontos, conforme o modelo utilizado pelo AMAQ (Quadro 2). Nesse caso, o padrão é considerado após declaração, pelo sujeito, da qualidade esperada. Esses padrões possuem caráter incremental em si mesmo, cuja avaliação da situação analisada se dá por meio da escala numérica disponibilizada. Eles estão organizados de modo a possibilitar a quantificação das respostas autoavaliativas, viabilizando a constituição de classificações gerais de qualidade. $O$ ponto 0 (zero) indica o não cumprimento ao padrão. Já o ponto 10 (dez) é a total adequação e os intervalos entre 0 (zero) e 10 (dez) são graus de conformidade ou atendimento da situação analisada em relação à qualidade desejada. 
Você está convidado a participar da avaliação sobre as ações relacionadas ao acompanhamento das condicionalidades da Saúde do Programa Bolsa Família. Para tanto, solicitamos que leia atentamente as questões abaixo e atribua uma pontuação, marcando um X no número escolhido. Para a escolha da pontuação, considere a escala abaixo:

Muito insatisfatório Muito satisfatório

012345678910

\section{Ações gerais relacionadas ao acompanhamento das famílias}

ESCALA DE PONTUAÇÃO

\begin{tabular}{|l|l|l|l|l|l|l|l|l|l|l|l|}
\hline 0 & 1 & 2 & 3 & 4 & 5 & 6 & 7 & 8 & 9 & 10 \\
\hline
\end{tabular}

As gestantes e nutrizes fazem a inscrição no pré-natal e comparecem às consultas na Unidade de Saúde, com o cartão da gestante, de acordo com o calendário mínimo preconizado pelo Ministério da Saúde?

ESCALA DE PONTUAÇÃO

\begin{tabular}{|l|l|l|l|l|l|l|l|l|l|l|}
\hline 0 & 1 & 2 & 3 & 4 & 5 & 6 & 7 & 8 & 9 & 10 \\
\hline
\end{tabular}

As gestantes e nutrizes participam de atividades educativas ofertadas pelas equipes de saúde sobre aleitamento materno e promoção da alimentação saudável?

\begin{tabular}{|l|l|l|l|l|l|l|l|l|l|l|}
\hline & \multicolumn{10}{c|}{ ESCALA DE PONTUAČ̃̃ } \\
\hline 0 & 1 & 2 & 3 & 4 & 5 & 6 & 7 & 8 & 9 & 10 \\
\hline
\end{tabular}

Os responsáveis pelas crianças menores de 7 anos mantêm em dia o calendário de vacinação, conforme preconizado pelo Ministério da Saúde?

ESCALA DE PONTUAÇÃO

\begin{tabular}{|l|l|l|l|l|l|l|l|l|l|l|}
\hline 0 & 1 & 2 & 3 & 4 & 5 & 6 & 7 & 8 & 9 & 10 \\
\hline
\end{tabular}

Os responsáveis pelas crianças menores de 7 anos realizam o acompanhamento do crescimento e desenvolvimento infantil por meio da vigilância alimentar e nutricional, conforme o calendário mínimo preconizado pelo Ministério da Saúde (1 vez por vigência)?

ESCALA DE PONTUAÇÃO

\begin{tabular}{|l|l|l|l|l|l|l|l|l|l|l|}
\hline 0 & 1 & 2 & 3 & 4 & 5 & 6 & 7 & 8 & 9 & 10 \\
\hline
\end{tabular}

Os responsáveis pelas crianças menores de 7 anos participam de atividades educativas ofertadas pelas equipes de saúde sobre promoção da alimentação saudável?

2.Ações relacionadas à atuação dos agentes comunitários de saúde ESCALA DE PONTUAÇÃO

\begin{tabular}{|l|l|l|l|l|l|l|l|l|l|l|l|}
\hline \multicolumn{1}{|c|}{ ESCALA DE PONTUAÇÃO } \\
\hline 0 & 1 & 2 & 3 & 4 & 5 & 6 & 7 & 8 & 9 & 10 \\
\hline
\end{tabular}

Você orienta as famílias beneficiárias sobre as condicionalidades da saúde do Programa Bolsa Família? ESCALA DE PONTUAÇÃO

\begin{tabular}{|l|l|l|l|l|l|l|l|l|l|l|}
\hline 0 & 1 & 2 & 3 & 4 & 5 & 6 & 7 & 8 & 9 & 10 \\
\hline
\end{tabular}

Você registra no mapa de acompanhamento as informações das condicionalidades da saúde juntamente com sua equipe de saúde (pelo menos 1 vez por vigência)? 


\begin{tabular}{|c|c|c|c|c|c|c|c|c|c|c|}
\hline \multicolumn{11}{|c|}{ ESCALA DE PONTUAÇÃO } \\
\hline 0 & 1 & 2 & 3 & 4 & 5 & 6 & 7 & 8 & 9 & 10 \\
\hline \multicolumn{11}{|c|}{$\begin{array}{l}\text { Você realiza a identificação das famílias que vivem em piores condições de moradia, educação, saneamentc } \\
\text { e alimentação, e que ainda não são beneficiadas do PBF? }\end{array}$} \\
\hline \multicolumn{11}{|c|}{ ESCALA DE PONTUAÇÃO } \\
\hline 0 & 1 & 2 & 3 & 4 & 5 & 6 & 7 & 8 & 9 & 10 \\
\hline \multicolumn{11}{|c|}{ Você orienta a família sobre a melhor forma de utilizar o recurso financeiro repassado pelo programa? } \\
\hline \multicolumn{11}{|c|}{ ESCALA DE PONTUAÇÃO } \\
\hline 0 & 1 & 2 & 3 & 4 & 5 & 6 & 7 & 8 & 9 & 10 \\
\hline \multicolumn{11}{|c|}{$\begin{array}{l}\text { Você conversa com os outros membros da equipe de saúde, com a comunidade e com outros setores do } \\
\text { governo sobre as possíveis ações do município para facilitar o acesso do beneficiário a programas de trans- } \\
\text { ferência de renda, bem como sobre outras formas de aumentar a renda familiar? }\end{array}$} \\
\hline \multicolumn{11}{|c|}{ ESCALA DE PONTUAÇÃO } \\
\hline 0 & 1 & 2 & 3 & 4 & 5 & 6 & 7 & 8 & 9 & 10 \\
\hline \multicolumn{11}{|c|}{$\begin{array}{l}\text { Você incentiva a comunidade no desempenho do seu papel cidadão na sociedade? } \\
\text { ESCALA DE PONTUAÇAO }\end{array}$} \\
\hline 0 & 1 & 2 & 3 & 4 & 5 & 6 & 7 & 8 & 9 & 10 \\
\hline Você & $a$ as & as sc & $i m p$ & ia c & oar & ăo & nse & unis & & \\
\hline
\end{tabular}

Quadro 2 - Instrumento utilizado na Avaliação do Programa Bolsa Família

Fonte: Adaptado com base em AMAQ-AB (2017).

Ao final da autoavaliação dos padrões foi possível conhecer a classificação do participante (ACS) para cada subdimensão nas categorias (Quadro 3): muito insatisfatório, insatisfatório, regular, satisfatório e muito satisfatório. Cada subdimensão representa um total de pontos que variam de acordo com o número de padrões de qualidade, sendo a estes atribuídos 10 (dez) pontos.

\section{SUBDIMENSÃO}

Ações gerais relacionadas ao acompanhamento das famílias

Ações relacionadas à atuação dos agentes comunitários de saúde

$$
\begin{array}{l|l|}
\text { Número de Padrões } & \multicolumn{2}{|c}{\text { Pontuação }} \\
\text { Mínima } & \text { Máxima }
\end{array}
$$

05

0

07

0 70

Quadro 3 - Subdimensões por Padrões de Qualidade

Fonte: Adaptado com base em AMAQ-AB (2017). 
A classificação da subdimensão quanto ao seu nível de qualidade foi feita por meio do cálculo dos percentuais dos pontos conquistados em relação ao total de pontos possíveis, associando o resultado observado à escala definida. O quadro 4 mostra a escala utilizada, que distribui, de maneira percentual, os pontos das subdimensões em 05 categorias. Para classificar a dimensão, foi utilizada a média ponderada entre as subdimensões. A classificação da dimensão consiste na média das avaliações de suas subdimensões, resultando em pontuação que varia entre os escores de 1 (um) a 5 (cinco).

\begin{tabular}{|l|c|c|c|c|c|}
\hline Classificação & $\begin{array}{c}\text { Muito } \\
\text { Insatisfatório }\end{array}$ & Insatisfatório & Regular & Satisfatório & Muito Satisfatório \\
\hline Porcentagem & $0-19,99$ & $20-39,99$ & $40-59,99$ & $60-79,99$ & $80-100$ \\
\hline Pontos & 1 & 2 & 3 & 4 & 5 \\
\hline
\end{tabular}

Quadro 4 - Classificação e Percentual das Subdimensões

Fonte: Adaptado com base em AMAQ-AB (2017).

Os sujeitos da prática, escolhidos pela sua distribuição no tecido social, foram os 15 (quinze) agentes comunitários de saúde das quatro equipes da Estratégia Saúde da Família no Bairro Nordeste, no Distrito Sanitário Oeste do Município de Natal (RN). Os critérios de inclusão foram: ser ACS; estar lotado na referida ESF; desejar espontaneamente participar da prática; realizar regularmente acompanhamento das condicionalidades da saúde do PBF.

O desenvolvimento da atividade e a construção dos dados ocorreram em meados de 2013, durante três momentos, contribuindo com o empoderamento atitudinal dos sujeitos frente às questões colocadas, de maneira a proporcionar aos agentes comunitários de saúde momentos adequados de reflexão, discussão sobre suas práticas, limites de atuação e possibilidades emergentes de mudanças.

No primeiro momento, ocorreu uma sensibilização e apresentação dos relatórios consolidados do Programa Bolsa Família, referentes ao ano anterior ( $1^{\mathrm{a}}$ e $2^{\mathrm{a}}$ vigências), contendo os resultados da análise documental de cobertura alcançada pelo município de Natal e pela Unidade de Saúde em foco. No segundo momento, foram realizadas a leitura e a aplicação do instrumento de autoavaliação; e no terceiro momento, a análise dos resultados. É importante destacar que, mesmo adaptando o uso de um instrumento previamente validado nacionalmente, as cinco primeiras aplicações aconteceram como testagem-piloto, de forma que, na ausência de necessidades de modificação da ferramenta, as demais aplicações seguiram conforme programado.

$\mathrm{Na}$ ocasião, foi ressaltada a importância da cultura de processos autoavaliativos e de monitoramento na identificação das potencialidades, fragilidades e estratégias de enfrentamento para a melhoria dos serviços e da satisfação do profissional no trabalho. Éconveniente esclarecer que o acompanhamento das condicionalidades do PBF na saúde, até o ano de 2013, no município, era realizado exclusivamente pelos ACS (considerados atores sociais relevantes para esta atividade) e que, a partir do ano seguinte, foram contratados estagiários do curso de nutrição para tal tarefa, que permanecem nessa função até o presente.

No que diz respeito aos aspectos éticos, a investigação seguiu as Diretrizes e Normas Regulamentadoras para pesquisa envolvendo seres humanos, aprovadas pelo Conselho Nacional de Saúde do Ministério da Saúde, conforme resolução n 466/12. Foram respeitadas as recomendações quanto aos esclarecimentos necessários a todos os integrantes, participação voluntária, assinatura do termo de consentimento livre e esclarecido, sigilo e guarda das informações, entre outras. 


\section{RESULTADOS}

Nesta seção, apresentaremos os resultados provenientes da análise documental e da aplicação do instrumento de autoavaliação proposto.

\subsection{A análise documental}

O Bairro Nordeste (cenário da prática) faz parte do Distrito Oeste de Natal (RN) e seus limites são: Rio Potengi (norte e oeste); bairro das Quintas (leste); bairro Bom Pastor (sul). Em relação à situação sanitária, 97,7\% das residências possuem abastecimento de água e 90,9\% do lixo é coletado (IBGE2010), assim como $99,9 \%$ dos domicílios possuem esgoto (CAERN-2010). Os dados do sistema de informação indicaram uma população cadastrada de 10.228 habitantes, sendo 5.457 do sexo feminino e 4.771 do sexo masculino, com renda média familiar em torno de um a três salários mínimos.

O território possui os seguintes equipamentos sociais: uma Associação dos Alcoólicos Anônimos; três Associações de Moradores; um Clube de Mães; um Grupo de Idosos; três escolas públicas; três escolas particulares; duas creches; uma emissora de rádio; quatro igrejas evangélicas e uma igreja católica. $\bigcirc$ bairro possui algumas áreas de poluição ambiental (contaminação do ar, água) devido a padarias, serrarias, borracharias, esgotos e lixo a céu-aberto; rios e mangue poluídos em contato direto com residências em duas microáreas; casa de prostituição; criação de animais; sucata, linha férrea e uma rodovia de intenso tráfego.

A Estratégia de Saúde da Família (ESF) foi implantada nesse bairro em outubro de 2003, e possui quatro equipes com áreas de abrangência divididas em cinco microáreas, cada uma com 05 ACS, sendo, entretanto, apenas 17 deles atuantes. Segundo análise dos dados coletados no Relatório Consolidado do PBF no período em questão, obtido no sistema de informação do Programa, no município de Natal (RN), na $1^{\text {a }}$ vigência do PBF existiam 36.278 famílias beneficiárias (perfil saúde), das quais apenas 13.287 foram acompanhadas. Esses dados representam um percentual de aproximadamente $37 \%$ de famílias acompanhadas. Já na $2^{a}$ vigência, existiam 40.097 famílias beneficiárias (perfil saúde), sendo apenas 12.146 acompanhadas, o que representa um percentual de aproximadamente $30 \%$ de acompanhamento das famílias. Em ambas as vigências, o índice de acompanhamento das famílias ficou muito abaixo da meta estabelecida para o Estado do Rio Grande do Norte, que é de $70 \%$ de acompanhamento.

No Distrito Sanitário Oeste, na $1^{\text {a }}$ vigência (Figura 1), existia 9.678 famílias beneficiárias, sendo que apenas 5.249 foram acompanhadas. Esse número representou um percentual de acompanhamento de $54 \%$. Já na $2^{a}$ vigência (Figura 2), o número de famílias beneficiárias era de 10.061, porém foram acompanhadas somente 4.790 famílias. Apesar desse número representar um acompanhamento maior em relação ao município (48\%), ainda se encontra abaixo da meta estabelecida.

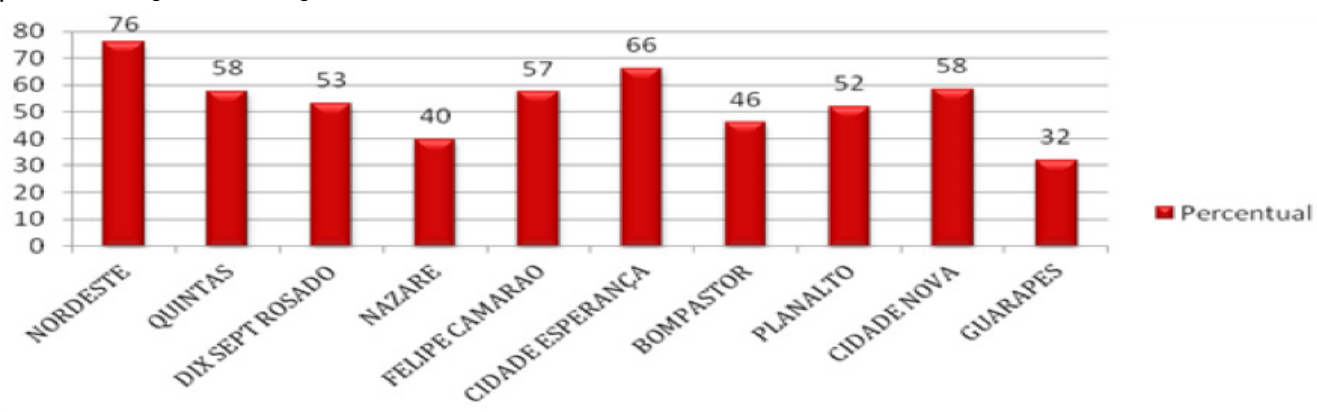

Figura 1 - Percentual de Acompanhamento do Programa Bolsa Família Distrito Sanitário Oeste (1 ${ }^{a}$ Vigência). 


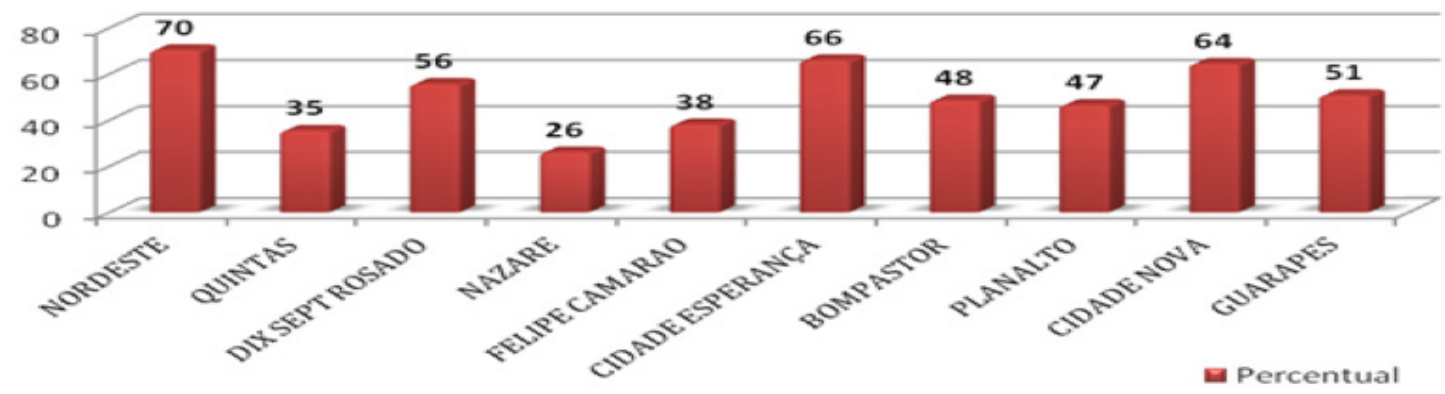

Figura 2 - Percentual de Acompanhamento do Programa Bolsa Família Distrito Sanitário Oeste (2ª Vigência).

Fonte: SAS/DAB/MS (2013).

Ainda em relação ao acompanhamento das condicionalidades da saúde, na Unidade de Estratégia Saúde da Família Bairro Nordeste, durante a $1^{\text {a }}$ Vigência, o acompanhamento das famílias ficou em torno de $76 \%$. A unidade possuía 402 famílias beneficiárias, das quais 305 foram acompanhadas pela unidade. $\mathrm{Na} 2^{\mathrm{a}}$ vigência, esses números foram de 434 famílias beneficiárias, destas, 305 famílias foram acompanhadas nas condicionalidades da saúde, o que gerou um percentual de $70 \%$ de acompanhamento. Nesse caso, o que chama a atenção é que, nas duas vigências, esse percentual está muito acima do índice atingido pelas demais Unidades de Saúde do município e do próprio Distrito Sanitário em que se localiza.

Nesse contexto, a USF do Bairro Nordeste mostrou um desempenho diferenciado do percentual de acompanhamento do referido município em relação às demais unidades de saúde, corroborando a existência de evidências dos impactos positivos na saúde das famílias atendidas pelo PBF. Isso ocorreu por meio da intensificação do acompanhamento das condicionalidades por parte das equipes de saúde, gerando maior acesso aos serviços, bem como destacando a importância dos agentes comunitários de saúde nesse processo (ALMEIDA; ALMEIDA; FERRANTE, 2015; CAMARGO; PAZELLO, 2014; COELHO; MELO, 2017).

\section{$3.2 \mathrm{O}$ instrumento de autoavaliação}

A autoavaliação das ações de acompanhamento das famílias e do desempenho dos ACS foi realizada mediante a somatória dos pontos de cada subdimensão. Foram calculados os percentuais dos pontos obtidos em relação ao total de pontos possíveis de cada subdimensão, relacionando os resultados com os percentuais definidos que, por sua vez, foram reclassificados por categorias, de acordo com uma escala de pontuação que serviu de base para a construção de uma média ponderada das subdimensões de cada participante. A essa média foi aplicada uma escala categórica de pontuação. O quadro 5 mostra os resultados consolidados finais dos instrumentos avaliativos aplicados.

\begin{tabular}{|c|c|c|c|c|}
\hline Subdimensão & \multicolumn{3}{|c|}{ Ações gerais relacionadas ao acompanhamento das famílias } \\
\hline Muito Insatisfatório & Insatisfatório & Regular & Satisfatório & Muito Satisfatório \\
\hline 1 ponto & 2 pontos & 3 pontos & 4 pontos & 5 pontos \\
\hline Subdimensão & & Ações relacionadas à atuação dos ACS & \\
& & & $X$ \\
\hline Total & \multicolumn{3}{|r|}{ Média da pontuação: $4+4=8 / 2=4$ (Satisfatório) } \\
\hline
\end{tabular}

Quadro 5 - Consolidação final da pontuação das subdimensões. 


\begin{tabular}{|c|c|c|c|c|c|c|c|c|c|}
\hline \multirow{2}{*}{\multicolumn{2}{|c|}{$\begin{array}{c}\text { Subdimensão } \\
\text { Muito Insatisfatório }\end{array}$}} & \multicolumn{8}{|c|}{ Ações gerais relacionadas ao acompanhamento das famílias } \\
\hline & & \multicolumn{2}{|c|}{ Insatisfatório } & \multicolumn{2}{|c|}{ Regular } & \multicolumn{2}{|c|}{ Satisfatório } & \multicolumn{2}{|c|}{ Muito Satisfatório } \\
\hline ACS & $\%$ & ACS & $\%$ & ACS & $\%$ & ACS & $\%$ & ACS & $\%$ \\
\hline - & $\begin{array}{c}0- \\
19,99\end{array}$ & - & $\begin{array}{c}20- \\
39,99\end{array}$ & 01 & $\begin{array}{r}40- \\
59,99\end{array}$ & 11 & $\begin{array}{c}10 \\
60- \\
79,99\end{array}$ & 03 & $\begin{array}{l}80- \\
100\end{array}$ \\
\hline \multicolumn{2}{|c|}{ Subdimensão } & \multicolumn{8}{|c|}{ Ações relacionadas à atuação dos ACS } \\
\hline \multicolumn{2}{|c|}{ Muito Insatisfatório } & \multicolumn{2}{|c|}{ Insatisfatório } & \multicolumn{2}{|c|}{ Regular } & \multicolumn{2}{|c|}{ Satisfatório } & \multicolumn{2}{|c|}{ Muito Satisfatório } \\
\hline ACS & $\%$ & ACS & $\%$ & ACS & $\%$ & ACS & $\%$ & ACS & $\%$ \\
\hline 政 & $\begin{array}{c}0- \\
19,99\end{array}$ & - & $\begin{array}{r}20- \\
39,99\end{array}$ & 02 & $40-$ & 08 & $60-$ & 05 & $\begin{array}{l}80- \\
100\end{array}$ \\
\hline
\end{tabular}

Quadro 6 - autoavaliação específica de cada subdimensão.

Fonte: Adaptado com base em AMAQ-AB (2017).

Diante disso, foi observado que na subdimensão Ações gerais relacionadas ao acompanhamento das famílias $73 \%$ dos participantes obtiveram desempenho satisfatório, enquanto que na subdimensão Ações relacionadas à atuação dos agentes comunitários de saúde o percentual de desempenho satisfatório foi de $53 \%$. Na consolidação das 02 subdimensões, obtidas a partir da média ponderada de análise, observamos que $20 \%$ dos participantes obtiveram classificação regular, 60\% classificação satisfatória e $20 \%$ classificação muito satisfatória.

$O$ instrumento de autoavaliação confirmou os resultados observados na análise documental dos relatórios consolidados do Sistema de Informação do PBF. As ESF mostraram percentuais de desempenho ainda muito discrepantes (desiguais), saltando aos olhos a ocorrência de algumas ilhas de excelência. Os resultados apontaram a necessidade de aprofundamento da discussão sobre os fatores que contribuem para o bom desempenho dos ACS da Unidade de Saúde da Família Bairro Nordeste, em relação às demais Unidades do Distrito Oeste de Natal (RN).

A literatura apresenta estudos pertinentes acerca da importância do acompanhamento intersetorial e, em diferentes contextos, das condicionalidades do PBF na saúde dos beneficiários, a exemplo das investigações de: Mariano e Carloto (2013), sobre a população negra; Cacciamali, Tatei e Batista
(2010), acerca do trabalho infantil; Moreira et al. (2012), sobre assistência social; Santos et al. (2014), no protagonismo do usuário beneficiário; Santos, Silva e Koller (2017), com a população ribeirinha; e Pires e Jardim (2014), na área rural. Entretanto, identificamos uma lacuna em relação a trabalhos sobre o uso de ferramentas para autoavaliação do acompanhamento dessas condicionalidades com características semelhantes ao instrumento apresentado neste artigo.

\section{CONCLUSÕES}

A atividade possibilitou identificar alguns desafios e/ou problemas críticos, orientando, assim, a definição de prioridades para seu enfrentamento. Alertou também para a relevância dos registros locais de acompanhamento para uma melhor organização do processo de trabalho das equipes da Estratégia Saúde da Família a fim de que a política social do PBF tenha o devido alcance aos usuários cadastrados.

Permitiu, desse modo, repensar o desenho das condicionalidades exigidas para o setor saúde na busca de critérios mais adequados à melhoria da qualidade dos serviços, indo além da simples existência do serviço ofertado. $\bigcirc$ artigo apresentou um instrumento pertinente de autoavaliação capaz de auxiliar a gestão local no monitoramento dessas condicionalidades na saúde, sendo etapa essencial para uma posterior 
testagem acerca do funcionamento do programa a nível municipal. Recomendamos como contribuição importante a disponibilização do instrumento desenvolvido para aplicação por outras equipes da ESF, para comparação e generalização dos resultados.

Cabe ressaltar que a vivência ainda contribuiu para aumentar o conhecimento dos atores, possibilitando a reflexão sobre seus processos de trabalho e o aprimoramento de habilidades e competências autoavaliativas, no complexo e indefinido campo de fronteiras entre políticas públicas focais e universais. Esta prática evidenciou a necessidade de complementação do estudo desses aspectos através de uma avaliação qualitativa, capaz de possibilitar a identificação de possíveis intervenções que possam ser aplicadas para as demais equipes do município, no intuito de subsidiar o planejamento das ações e tomadas de decisões. 


\section{REFERÊNCIAS}

ALMEIDA, F. B.; ALMEIDA, L. M. M. C.; FERRANTE, V. L. S. B.

Avaliação do Programa Bolsa Família na segurança alimentar das famílias rurais do município de Rio Verde, GO: efeitos, entraves e diferenciações. Interações, Campo Grande, v. 16, n. 2, p. 277-287, dez. 2015.

BRASIL. Lei ordinária n 10.836 de 9 de janeiro de 2004: Cria o Programa Bolsa Família e dá outras providências. 2004. Disponível em: < http://www.planalto.gov.br/ccivil_03/_ato2004-2006/2004/lei/ 110.836.htm>. Acesso em: 15 ago. 2017.

Ministério do Desenvolvimento Social e Combate à Fome. Guia para acompanhamento das condicionalidades do Programa Bolsa Família volume I. Guias e Manuais. Brasília, 2010.

- Ministério da Saúde. Coordenação-Geral de Alimentação e Nutrição. Organização Pan-Americana da Saúde. Observatório de Políticas de Segurança Alimentar e Nutrição. Universidade de Brasília. Curso de auto aprendizado. Matriz de Interfaces do Programa Bolsa Família na Saúde. Brasília, 2012.

- Ministério da Saúde. Secretaria de Atenção à Saúde.

Departamento de Atenção Básica. Autoavaliação para melhoria do acesso e da qualidade da atenção básica: AMAO. Brasília: Ministério da Saúde, 2017.

CABRAL, M. J. et al. Perfil socioeconômico, nutricional e de ingestão alimentar de beneficiários do Programa Bolsa Família. Estud. av., v. 27, n. 78, p. 71-87, 2013.

CACCIAMALI, M. C.; TATEI, F.; BATISTA, N. F. Impactos do Programa Bolsa Família federal sobre o trabalho infantil e a frequência escolar. Rev. econ. contemp., v. 14, n. 2, p. 269-301, ago. 2010.

CAMARGO, P. C., PAZELLO, E. T. Uma análise do efeito do programa bolsa família sobre o desempenho médio das escolas brasileiras. Econ. Apl., v. 18, n. 4, p.623-640, dez. 2014.

CAMPARA, J. P.; VIEIRA, K. M.; POTRICH, A .C. G. Satisfação Global de Vida e Bem-estar Financeiro: desvendando a percepção de beneficiários do Programa Bolsa Família. Rev. Adm. Pública, v. 51, n. 2, p. 182-200, mar. 2017.

CARVALHO, A. T.; ALMEIDA, E. R.; JAIME, P. C. Condicionalidades em saúde do programa Bolsa Família - Brasil: uma análise a partir de profissionais da saúde. Saude soc., v. 23, n. 4, p.1370-1382, dez. 2014. 
COELHO, P. L.; MELO, A. Impacto do Programa "Bolsa Família" sobre a qualidade da dieta das famílias de Pernambuco no Brasil. Ciênc. saúde coletiva, v. 22, n. 2, p. 393-402, fev. 2017.

GONÇALVES, G. Q.; MENICUCCI, T. M. G.; AMARAL, E. F. L. Diferencial educacional entre beneficiários e não beneficiários do Programa Bolsa Família. Cad. Pesqui., v. 47, n. 165, p. 770-795, set. 2017.

MARIANO, S. A.; CARLOTO, C. M. Aspectos diferenciais da inserção de mulheres negras no Programa Bolsa Família. Soc. estado., v. 28, n. 2, p. 393-417, ago. 2013.

MOREIRA, N. C. et al. Empoderamento das mulheres beneficiárias do Programa Bolsa Família na percepção dos agentes dos Centros de Referência de Assistência Social. Rev. Adm. Pública, v. 46, n. 2, p. 403-423, abr. 2012.

PIRES, F. F.; JARDIM, G. A. S. Geração bolsa família escolarização, trabalho infantil e consumo na casa sertaneja (Catingueira/PB). Rev. bras. Ci. Soc., v. 29, n. 85, p. 99-112, jun. 2014.

RIBEIRO, F. G.; SHIKIDA, C.; HILLBRECHT, R. O. Bolsa Família: Um survey sobre os efeitos do programa de transferência de renda condicionada do Brasil. Estud. Econ., v .47, n. 4, p. 805-862, dez. 2017.

SANTOS, M. C. M. et al. A voz do beneficiário: uma análise da eficácia do Programa Bolsa Família. Rev. Adm. Pública, v. 48, n. 6, p. 1381-1405, dez. 2014.

SANTOS, T. M.; SILVA, S. S. C.; KOLLER, S. H. Avaliação de Beneficiárias Ribeirinhas da Amazônia sobre o Programa Bolsa Família. Psic.: Teor. e Pesq., v. 33, 2017.

SOUZA, X. R.; MARIN, A. H. Intervenção com famílias em descumprimentos das condicionalidades do Programa Bolsa Família. Saude soc., v. 26, n. 2, p. 596-605, jun. 2017.

VASCONCELOS, A. M. et al. Programa Bolsa Família e Geração "Nem-Nem": Evidências para o Brasil. Rev. Bras. Econ., v. 71, n. 2, p. 233-257, Jun. 2017. 